\title{
Lock-in Amplifier as a Sensitive Instrument for Biomedical Measurement : Analysis and Implementation
}

\author{
Y.Abd Djawad ${ }^{\star 1}$, J. Kiely², P. Wraith ${ }^{2}$, and R. Luxton ${ }^{2}$ \\ ${ }^{1}$ State University of Makassar, Indonesia \\ ${ }^{2}$ University of the West of England, United Kingdom \\ Jl. AP Pettarani, Makassar, Indonesia, telp:0411-865894 \\ *Corresponding author, e-mail: yasser.djawad@unm.ac.id
}

\begin{abstract}
A measuring instrument plays important role in the biomedical measurement since the biological process in living organism generates very weak signal. Therefore, a reliable and sensitive measuring instrument is needed. In this study, a lock-in amplifier was analysed and tested. This paper presents an experiment to investigate the lock-in amplifier for biomedical measurement. An experiment using RC (resistor capacitor) tissue model to measure the voltage change related to impedance change was performed using a lock-in amplifier to evaluate the accuracy of the lock-in amplifier. Three different values of the capacitor in the $R C$ tissue model were applied regarding to simulate small impedance changes. The measurement results were compared with the theoretical calculation and an impedance measurement system. An error analysis was conducted to investigate the accuracy of the measurement. The comparison result showed that impedance measurement using lock-in amplifier is an effective technique, which could able to measure very small voltage regarding impedance change in the $R C$ tissue model.
\end{abstract}

Keywords: biomedical, RC tissue model, impedance measurement, lock-in amplifier

Copyright (C) 2014 Institute of Advanced Engineering and Science. All rights reserved.

\section{Introduction}

A Lock-in Amplifier (LIA) is a measuring instrument that measures a signal which has similar frequency and phase as the reference signal. The LIA can be used to measure a very small signal (nano volts) and is able to ignore any signals that are not synchronized with it, which may be a thousand times larger. The LIA consists of 5 blocks; a signal amplifier, a phase shifter to provide a reference signal, a phase sensitive detector (PSD) or multiplier, a low pass filter (LPF) and a DC amplifier, as shown in Figure 1. An input signal is connected to the amplifier. A second signal is connected to the phase shifter as a reference signal. The output is a DC voltage which is proportional to the amplitude of the signal being measured. The reference signal must be similar to the source signal to "lock" the signal to the frequency of interest. The LIA has been used in many applications such as measurement of signal to noise ratio of photothermal signals [1], chemistry experiments using diode laser [2], in a ring laser gyroscope [3] and nanoelectromechanical systems [4].

In biomedical research, the LIA is usually used in Impedance spectroscopy (IS) technique. IS is a characterization method of materials to obtain its electrical properties using electrodes [5]. IS can be divided into two categories, electrochemical impedance spectroscopy (EIS) and other techniques. EIS engages measurement and analysis of ionic conduction in the material. EIS is used also to study of fuel cells, rechargeable batteries, and corrosion. The resting category of impedance spectroscopy is applied to study electrical characteristic of dielectric material, solid or liquid non conductors, in which electronic conduction strongly in the majority[6]. IS has been also applied in biomedical research areas $[7,8,9,10,11]$. IS normally involves two electrodes, counter electrode (CE) and detecting electrode (DE). The sensor detects the change of resistance

Received June 19, 2014; Revised August 3, 2014; Accepted August 18, 2014 


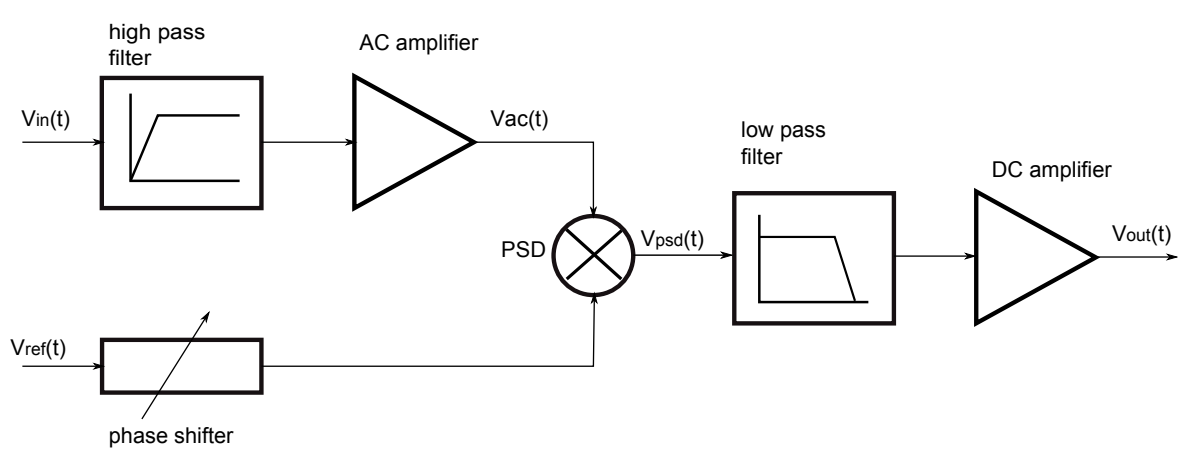

Figure 1. Block diagram of lock-in amplifier that consists of signal amplifier, phase shifter, PSD and low pass filter

and capacitance of the material above or between the sensor. The basic idea is to apply an input signal via $C E$ and gather the result on DE. These two electrodes with certain distance behaves like a capacitor when small AC voltage applied to the sensor. Signals with a range frequency are applied to the circuit to analyse the characteristics of the material. An example of developed of IS technique is Electric Cell-substrate Impedance Sensing (ECIS). The technique provides a very useful and elegant approach for the study of cell spreading, morphology and micromotion. Thefore, ECIS could be used for drug screening and toxicology studies in the animal testing [12]. The principle of ECIS is based on Ohm's law that the adhered cell can be treated as an equivalent of an RC circuit. ECIS uses electrodes that are coated by protein to provide a surface for cell attachment and spreading. When the cells begin to spread on the electrode surface, the cell layer starts to affect the electromagnetic field between the two electrodes since the adhered cells behave like insulating particles due to their plasma membrane [13]. This small electromagnetic field change on the electrode surface modifies the impedance of the capacitance component. Therefore, several researchers $[12,14,15]$ have used ECIS to predict the impedance of the cells and enable direct monitoring of impedance changing of the cells using the LIA as measuring instrument.

In this study the LIA has been choosen since it has some advantages like it can be build with low cost, very sensitive and can remove noise. A RC tissue model was used to replace the original cell tissue. The measurement was performed by connecting the LIA parallel with the RC tissue model. This RC tissue model is based on an approximation of RC values of human erythrocyte [16]. Rd characterizes the dielectric of the electrode/electrolyte. Rc is the cell resistance and the capacitor $C$ in this circuit represents the cell tissue. The LIA output was read by a PC Oscilloscope and the manual impedance calculation was conducted based on the LIA voltage output.

\section{Research Methodology}

\subsection{A trigonometry perspective of the LIA}

Presumably a signal with a frequency $f_{1}$ and phase of $\theta_{1}$ is applied to input channel. It is assumed that signal has a very low amplitude and it must be amplified with gain $G_{a c}$. The output of the amplifier is a signal with a specific gain, namely :

$$
V_{a c}(t)=G_{a c} V_{o} \cos \left(w_{1} t+\theta_{1}\right)
$$

In addition, a reference signal that has a frequency $f_{2}$ and $\theta_{2}$ is given as a multiplicand of the output signal from amplifier, the signal is :

$$
V_{\text {ref }}(t)=E_{o} \cos \left(w_{2} t+\theta_{2}\right)
$$

The product result of the amplifier output signal and the reference signal is an identity product of these two signals and can be expressed as : 


$$
\begin{array}{r}
V_{p s d}(t)=\frac{1}{2} G_{a c} V_{o} E_{o}\left(\cos \left[\left(w_{1}+w_{2}\right) t+\left(\theta_{1}+\theta_{2}\right)\right]+\right. \\
\left.\cos \left[\left(w_{1}-w_{2}\right) t+\left(\theta_{1}-\theta_{2}\right)\right]\right)
\end{array}
$$

Equation 3 shows that the product of two sinusoidal signals, which have different frequencies, produces two sinusoidal signals with different frequencies.

$$
\begin{aligned}
& V_{p s d 1}(t)=\frac{1}{2} G_{a c} V_{o} E_{o}\left(\cos \left[\left(w_{1}+w_{2}\right) t+\left(\theta_{1}+\theta_{2}\right)\right]\right) \\
& V_{p s d 2}(t)=\frac{1}{2} G_{a c} V_{o} E_{o}\left(\cos \left[\left(w_{1}-w_{2}\right) t+\left(\theta_{1}-\theta_{2}\right)\right]\right)
\end{aligned}
$$

If Equation 3 is applied to a LPF that has a frequency cut off smaller than $\left(w_{1}+w_{2}\right)$, the high frequency component (Equation 4 ) is attenuated and the low frequency component (Equation 5 ) is passed the LPF and the final result is sum of two sinusoidal signals with different frequencies.

When the frequency and phase of the input signal and the reference signal are similar, the Equation 3 becomes :

$$
V_{p s d}(t)=\frac{1}{2} G_{a c} V_{o} E_{o}[1+\cos (2 w t+2 \theta)]
$$

From Equation 6, it reveals that there are two signal components, AC and DC. The amplitude of the output signal is a half of the input signal and the frequency of $A C$ component is twice of the amplified input signal. When a LPF which has frequency cut off smaller than frequency of AC signal is applied to the Equation 6, the AC signal is attenuated by the LPF. The result is the sum of the DC signal and the attenuated AC signal which is a ripple DC signal.

Equation 3 demonstrates that if the frequencies and the phases of the input and reference signal are equal, the DC component is maximum since it produces zero phase different. If the input signal and the reference signal have different phase, the output signal does not reach maximum output which half of the amplified input signal. It concludes that the LIA only measures signals that have same frequency and phase as reference signal.

\subsection{Instrumentation}

For testing the LIA, a RC tissue model was used as shown in Figure 2. The RC tissue model consists of two fixed carbon resistors $22 \mathrm{Ohm}$ and a ceramic capacitor as a cell tissue which has three values $0.01 \mu F, 0.1 \mu F$ and $1 \mu F$. These values of capacitor were chosen to analyse the accuracy of the LIA when small impedance of the RC tissue model is changed.

To conduct the impedance measurement, the LIA was used. The high-pass filter (HPF) was set to have a frequency cut off $0.7 \mathrm{~Hz}$ and the low pass filter (LPF) was set to have a frequency cut off $16 \mathrm{~Hz}$. The HPF was used to remove the DC offset of the sensor. This avoids DC offset driving the chip into saturation mode. while the LPF was used to remove the noise and suppressed the AC component of the output signal. The frequency cut-off was chosen based on the interest of the signal. A frequency cut-off of $0.7 \mathrm{~Hz}$ was selected as this is sufficiently high to remove the DC component of the input signal. A frequency cut-off of $16 \mathrm{~Hz}$ was chosen based on the observation during experiment, since it provides smoothest output of DC signal. To construct a voltage divider, the RC tissue model was connected in series with a $1.5 \mathrm{KOhm}$ resistor. The $1.5 \mathrm{KOhm}$ resistor was designated to allow the majority of the voltage to be dropped across it and leave only very small portion of voltage to be dropped across the RC tissue model. An AC voltage with amplitude of $250 \mathrm{mV}$, from PICOScope (PICOScope is a real time PC-based digital oscilloscope, which has function of digital storage oscilloscope, meter and data logger, spectrum analyser and signal generator), and reference signal of the LIA with frequency range from $10 \mathrm{~Hz}$ - $1 \mathrm{MHz}$ were applied to the circuit. The LIA was connected parallel with the RC tissue model to detect small voltage drop across the RC tissue model. LIA output was measured by PicoScope. At a specific frequency value, the output amplitude of the LIA was recorded and the impedance 
was calculated to allow a comparison between theoretical values and $\mathrm{C} 60$ (impedance measuring instrument made by Cypher instruments Ltd) output.

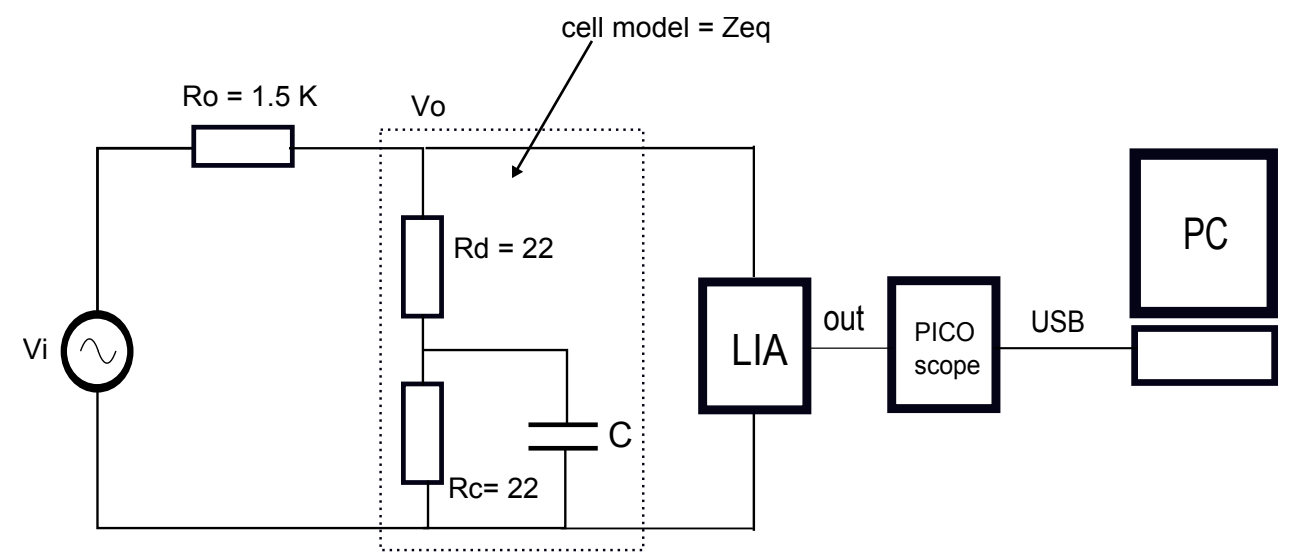

Figure 2. The circuit diagram of measurement system with the RC tissue model

\section{Result and Analysis}

\subsection{Experimental Results}

Theoretically, the cell impedance can be defined by following equations :

$$
\begin{gathered}
Z_{e q}=R_{d}+\left(\frac{R_{c}}{1+j \omega R_{c} C}\right) \\
Z_{e q}=R_{d}+\left(\frac{R_{c}}{1+j \omega R_{c} C} \cdot \frac{1-j \omega R_{c} C}{1-j \omega R_{c} C}\right) \\
Z_{e q}=R_{d}+\left(\frac{R_{c}-j \omega R_{c}^{2} C}{1+\omega^{2} R_{c}^{2} C^{2}}\right)
\end{gathered}
$$

and can be represented in the rectangular form as :

$$
Z_{e q}=\left(R_{d}+\frac{R_{c}}{1+\left(\omega C R_{c}\right)^{2}}\right)-j \frac{\omega C R_{c}^{2}}{1+\left(\omega C R_{c}\right)^{2}}
$$

in the polar form can be represented as follow :

Magnitude :

$$
\left|Z_{e q}\right|=\frac{\sqrt{\left(R_{d}+R_{c}+\omega^{2} R_{c}^{2} R_{d} C^{2}\right)^{2}+\left(\omega R_{c}^{2} C\right)^{2}}}{1+\left(\omega C R_{c}\right)^{2}}
$$

Phase:

$$
\theta=-\arctan \left(\frac{\omega C R_{c}^{2}}{R_{d}+R_{c}+\omega^{2} R_{c}^{2} R_{d} C^{2}}\right)
$$

When the frequency is very low, the imaginary part of Equation 8 is relatively low. As the imaginary part of cell impedances retains only a minor value, the impedance of the cell model is approximately equal to $R c+R d$. When the frequency gradually increases, the imaginary part also slowly increases until reaching a maximum at a specific frequency and then steadily decreases to zero again. Thus, the values of the $\mathrm{RC}$ tissue model provide very small effect to the whole impedance as shown in Figure 3.

Figure 4 shows the impedance change when the capacitor is changed. It shows that the impedance change is relatively small. The graph revealed that the impedance change between 


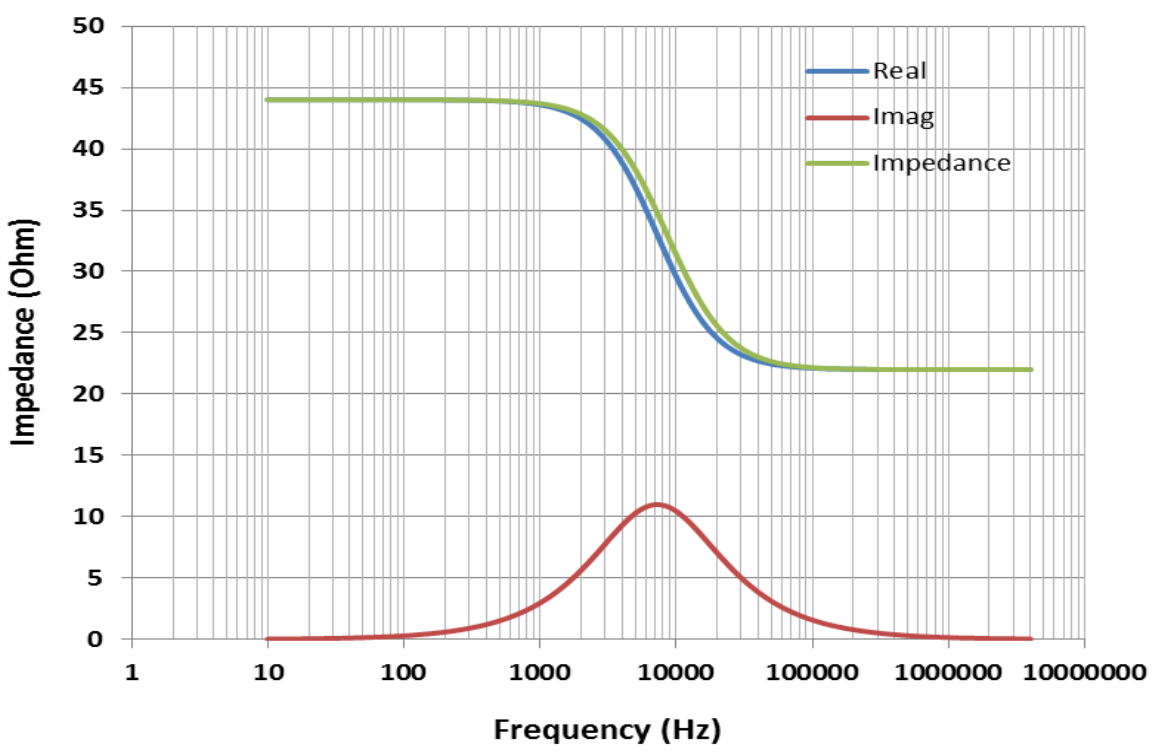

Figure 3. Contribution of imaginary part to the impedance of the RC tissue model with the capacitor of $1 \mu \mathrm{F}$

capacitor $0.01 \mu \mathrm{F}$ and $0.1 \mu \mathrm{F}$ is very small which is less than $0.1 \mathrm{Ohm}$. While the impedance change for capacitors $0.1 \mu \mathrm{F}$ and $1 \mu \mathrm{F}$ is exponentially increased. Similar result is happened with capacitor values of $0.01 \mu \mathrm{F}$ and $1 \mu \mathrm{F}$. The comparison of impedance change was limited until frequency around $3.3 \mathrm{kHz}$ since this is the smallest frequency cut off among three values of capacitor. Above this frequency, the impedance change increased because the smaller the capacitor value the bigger the frequency cut off.

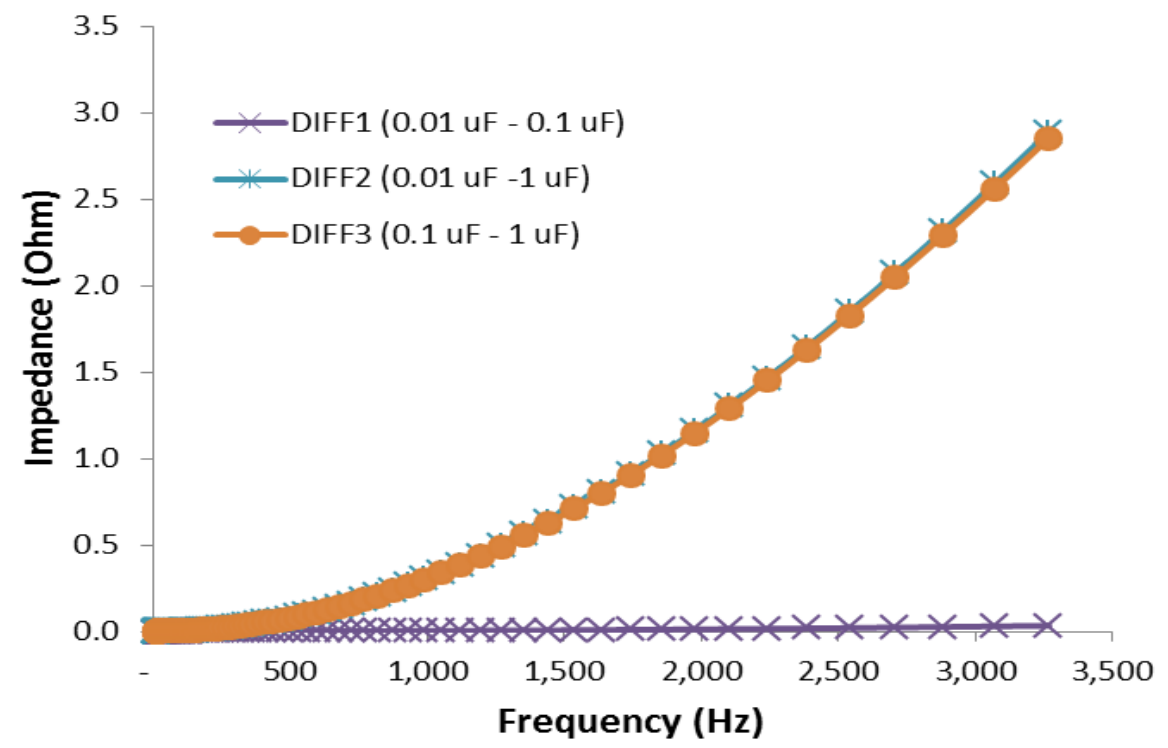

Figure 4. Impedance change as the capacitor values change until frequency cut off around 3.3 $\mathrm{kHz}$

Using the LIA voltage output, the impedance calculation is performed across a voltage divider as follows : 


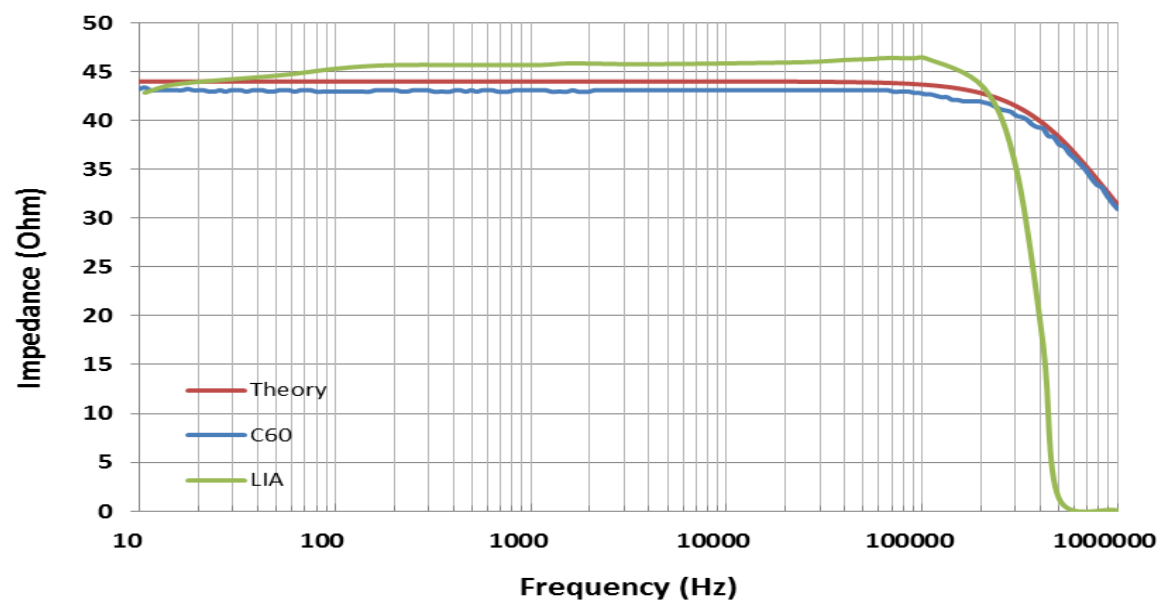

(a)

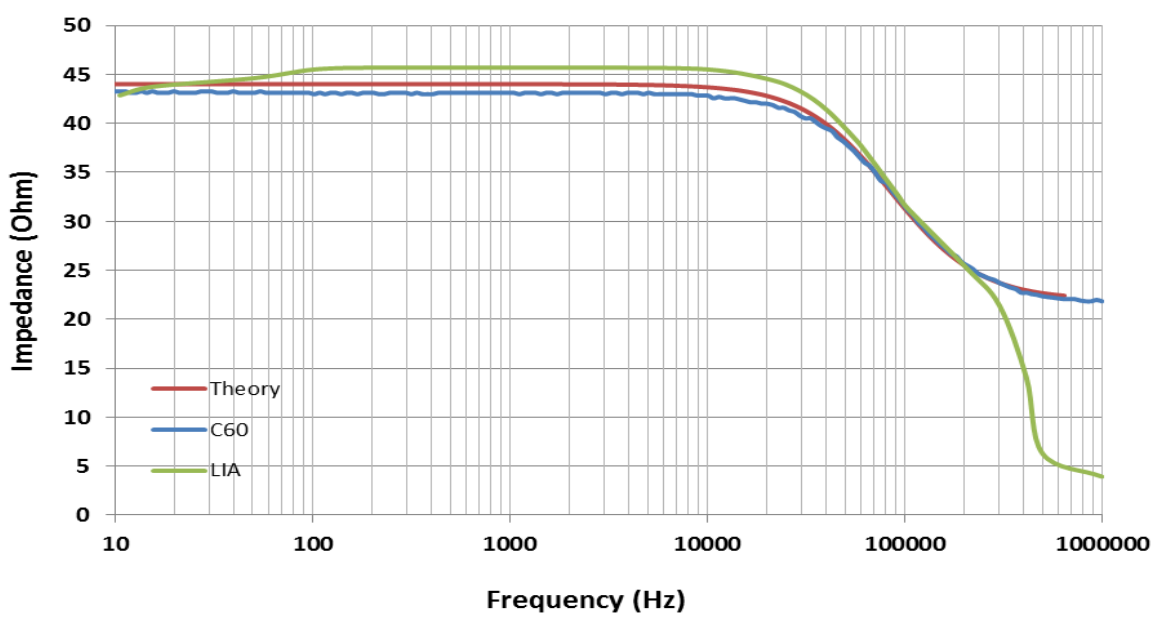

(b)

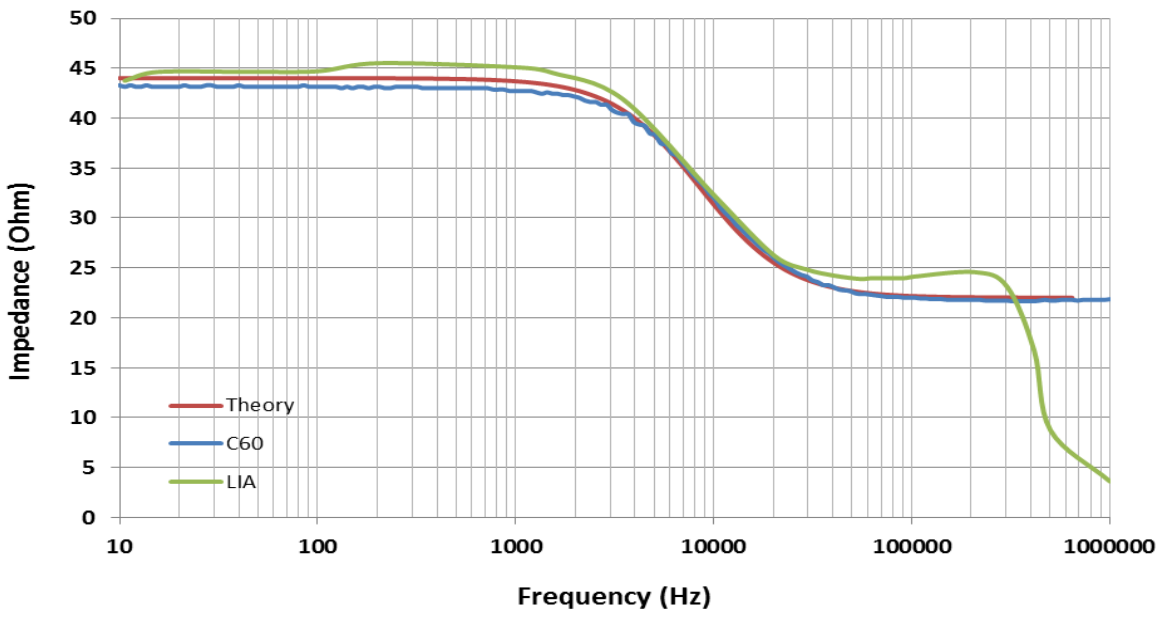

(c)

Figure 5. Comparison of the impedance measurement of the $\mathrm{RC}$ tissue model with capacitor of (a) $0.01 \mu \mathrm{F}$ (b) $0.1 \mu \mathrm{F}$ (c) $1 \mu \mathrm{F}$ 


$$
Z_{e q}=\frac{V_{o} R_{o}}{V_{i}-V_{o}}
$$

From Figure 5, it shows that the LIA performs adequate measurement compared with theoretical calculation and measurement using the C60 impedance measuring instrument. It also demonstrates that the LIA has limited measurement of approximately $210 \mathrm{KHz}$, as beyond this frequency the voltage decreases rapidly and provides less accurate results. The figures illustrate that the measurement of the LIA provides results close to the theoretical and the C60 output in the frequency range from $10 \mathrm{~Hz}-100 \mathrm{~Hz}$. Above this frequency, the impedance is slightly increased. Meanwhile at the slope, the measurements from the LIA provides improved outcome. It also can be observed that when the capacitor values are increase 10 times, the frequency cut off decreased approximately 10 times compared with previous frequency cut-off.

\subsection{Error analysis}

In this study, an error analysis was performed. The error analysis was conducted in the range frequency of $0-210 \mathrm{kHz}$ since this range is the limited frequency range of the LIA. Beyond this frequency range the measurement provide unacceptable results. In the error analysis, the relative error and standard error were used and defined as follows :

$$
\begin{gathered}
\delta=\frac{x_{o}-x}{x} \\
\sigma=\frac{S D}{\sqrt{N}}
\end{gathered}
$$

Where $x_{o}$ is the measurement value, $x$ is the theoretical value, $S D$ is the standard deviation of measurement and $N$ is the number of data in the measurement. Figure 6a illustrates the relative error graph of the LIA. It shows that relative errors of measurement using capacitor 0.01 $\mu \mathrm{F}$ are linearly increased from $4 \%$ until $100 \mathrm{kHz}$ and steadily decreases until frequency $210 \mathrm{kHz}$. While measurement using capacitor $0.1 \mu \mathrm{F}$, the graph shows that the relative errors are almost constant until frequency $30 \mathrm{kHz}$ and at this point the values are decreased linearly until frequency $100 \mathrm{kHz}$ and almost constant above $100 \mathrm{kHz}$. Different result showed by the measurement of 1 $\mu \mathrm{F}$. A spike of relative error showed at frequency $1.5 \mathrm{kHz}$. Above this frequency, the measurement showed constant values and increased until $210 \mathrm{kHz}$. While, Figure $6 \mathrm{~b}$ depicted the relative error graph of $\mathrm{C} 60$ measurement. The graph shows constant relative error for measurement using capacitor $0.01 \mu \mathrm{F}$ until around $60 \mathrm{kHz}$. The error rises above $60 \mathrm{kHz}$ and almost constant above $100 \mathrm{kHz}$ which is around $2 \%$. The measurement using capacitor $0.1 \mu \mathrm{F}$ provided better relative error which around $2 \%$ below $50 \mathrm{kHz}$ and constantly $0.5 \%$ above $50 \mathrm{kHz}$. Similar with the LIA measurement, the result of using capacitor $1 \mu \mathrm{F}$ provides a spike of relative error showed at 1.5 $\mathrm{kHz}$ and decreased slowly until $50 \mathrm{kHz}$ and after this frequency the result slowly rises until 210 $\mathrm{kHz}$.

The relative errors of both measurements presented varying relative error. The graphs depicted that relative error mean of $\mathrm{C60}$ is slightly better than the LIA, where the differences about 1-3\%. However, Table 1 describes the standard error of two measurements. It can be seen from Table 1, that both measurement results provide similar trend which as the capacitor value is increased the standard error is also rise. The error rises with margin about 1.5 as the capacitor value increases 10 times.

Table 1. Standard error of measurement

\begin{tabular}{|c|c|c|c|}
\hline$\sigma$ & $0.01 \mathrm{uF}$ & $0.1 \mathrm{uF}$ & $1 \mathrm{uF}$ \\
\hline LIA & 0.34 & 1.85 & 3.01 \\
\hline C60 & 0.10 & 1.61 & 3.00 \\
\hline
\end{tabular}




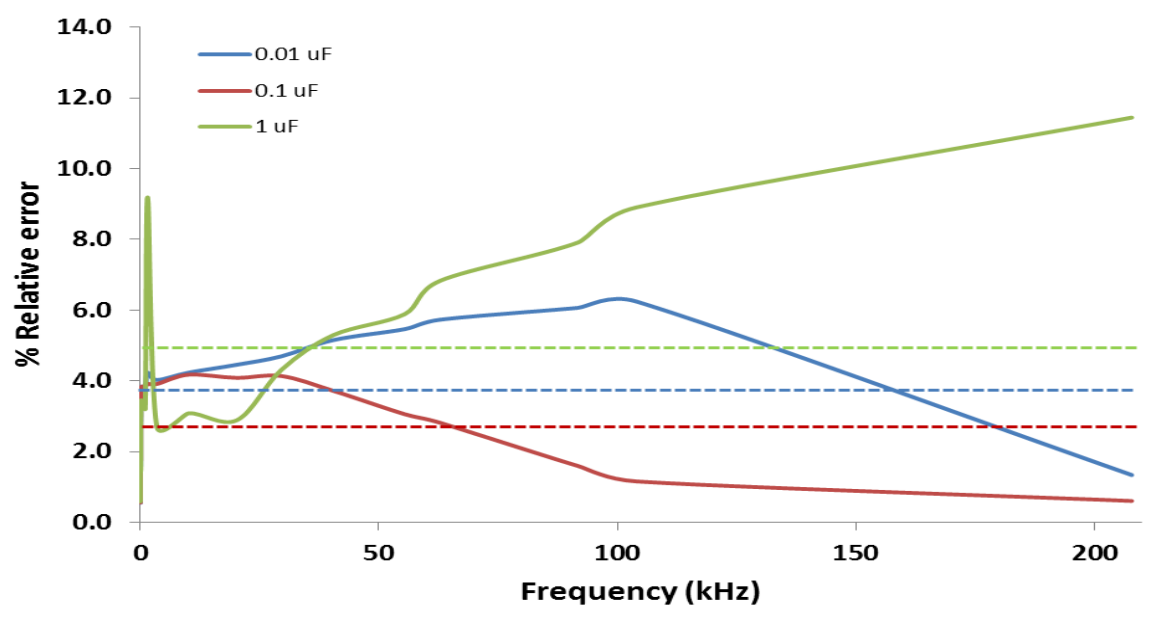

(a)

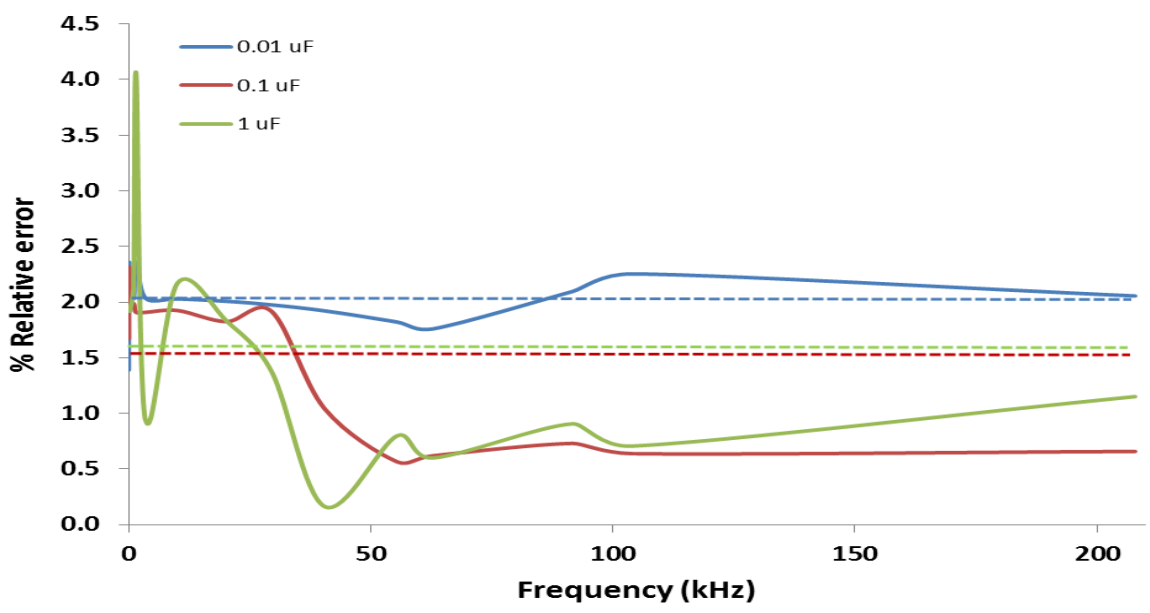

(b)

Figure 6. Standard error of measurement using (a) the LIA (b) C60. Dotted lines are mean of the standard error

\section{Conclusion}

The theory of lock-in amplifier has been reviewed and an experiment to measure the $\mathrm{RC}$ tissuel model using the LIA has been performed. The main idea of the LIA was described mathematically to gain deep understanding about how the LIA works and to observe the characteristics of the circuit. An experiment of impedance measurement of $R C$ tissue model using difference values of capacitor has been performed. The experiment revealed that the LIA was able to detect small voltage change in the $\mathrm{RC}$ tissue model as the impedance change. The comparison of the LIA with theoretical calculation and impedance measurement (C60) showed that measurement using the LIA provides an accurate measurement result which able to detect small impedance change. This ability is shown by the error analysis, where the relative error of the LIA measurement is relatively small. Therefore, this study showed that LIA can be used as an alternative low cost and an effective measuring instrument for biomedical measurement.

\section{References}

[1] A. Mandelis, "Signal-to-noise ratio in lock-in amplifier synchronous detection: A generalized communications systems approach with applications to frequency, time, and hybrid (rate 
window) photothermal measurements," Review of Scientific Instruments, vol. 65, pp. 33093323, 1994.

[2] J. Whitten, "Blue diode lasers:new opportunities in chemical education," J. Chemical Education, vol. 78, pp. 1096-1100, 2001.

[3] B. Wang, W. Zhang, Z. Wang, and P. Zhu, "Lock-in amplifier technology in laser gyroscope north finder of constant rate biasing," Mathematical Problems in Engineeringl, vol. 2013, pp. 1-11, 2013.

[4] Y. Yang, C. Callegari, X. Feng, and K. Ekinci, "Zeptogram-scale nanomechanical mass sensing," Nano Lett., vol. 6, pp. 583-586, 2006.

[5] J. Macdonald, Impedance Spectroscopy Theory, Experiment and Applications, 2nd ed. Wiley-Interscience, 2005.

[6] _ - "Impedance spectroscopy," Annals of Biomedical Engineering, vol. 20, pp. 289-305, 1992.

[7] C. Xiao, B. Lachance, G. Sunahara, and J. Luong, "Assesment of citotoxicity using cellsubstrate impedance sensing:concentration and time response function approach," Analytical Chemistry, vol. 74, pp. 5748-5753, 2002.

[8] T. Houssin, J. Follet, A. Follet, E. Dei-Cas, and V. Senez, "Label-free analysis of waterpolluting parasite by electrochemical impedance spectroscopy," Biosensors and Bioelectronics, vol. 25, pp. 1122-1129, 2010.

[9] M. McCoy and E. Wang, "Use of electric cell-substrate impedance sensing as a tool for quantifying cytopathic effect in influenza a virus infected mdck cells in real-time," Journal of Virulogical Methods, vol. 130, pp. 157-161, 2005.

[10] I. Ivanov, "Impedance spectroscopy of human erythrocyte membrane: Effect of frequency at the spectrin denaturation transition temperature," Bioelectrochemistry, vol. 78, pp. 181-185, 2010.

[11] L. Arias, C. Perry, and L. Yang., "Real-time electrical impedance detection of cellular activities of oral cancer cells," Biosensors and Bioelectronics, vol. 25, pp. 2225-2231, 2010.

[12] J. Luong, "An emerging impedance sensor based on cell-protein interactions: Applications in cell biology and analytical biochemistry," Analytical Letters, vol. 36, pp. 3147-3164, 2003.

[13] C. Keese, N. Karra, B. Dillon, A. Goldberg, and I. Giaever, "In vitro mol." Toxicol, vol. 11, pp. 83-192, 1998.

[14] L. Wang, L. Wang, H. Yin, W. Xing, Z. Yu, M. Guo, and J. Cheng, "Real time, label-free monitoring of the cell cycle with a cellular impedance sensing chip," Biosensors and Bioelectronics, vol. 25, pp. 990-995, 2010.

[15] C. Xiao and J. Luong, "A simple mathematical model for electric cell-substrate impedance sensing with extended applications," Biosensors and Bioelectronics, vol. 25, pp. 1774-1780, 2010.

[16] J. Bao, C. Davis, and R. Schmukler, "Frequency domain impedance measurements of erythrocytes," Biophysics, vol. 61, pp. 1427-1434, 1992. 